\title{
Performance and Application of an Aberration Corrected Analytical Electron Microscope with a Cold Field Emission Gun
}

\author{
Y. Kohno, E. Okunishi, I. Ishikawa, T. Tomita, T. Kaneyama and Y. Kondo \\ JEOL Ltd., Tokyo, Japan
}

A spherical aberration corrected electron microscope can provide smaller probes at higher current. These probes improve imaging $[1,2]$ and analysis of local fine structure using energy dispersive Xray spectrometry (EDS) or electron energy loss spectroscopy [3, 4]. The performance for imaging and analysis with the corrected microscope depends largely on the electron source. Once the spherical aberration is corrected, the probe size and probe current are limited by the chromatic aberration originates from the energy spread and the brightness of the source. In this respect, a cold field emission gun (FEG) is better than a Schottky gun. In fact, the cold FEG has been known as an electron source providing a small energy spread as well as high brightness. As shown in Fig. 1, we have successfully developed a new stable cold field emission gun (FEG) for a $200 \mathrm{kV}$ aberration corrected analytical electron microscope: JEM-ARM200F.

The cold FEG, however, has a difficulty in achieving the stability of the emission. The decrease in and the fluctuation of the emission current made the cold FEG difficult to handle. The main cause of this instability is the change in the work function due to adsorption and desorption of residual gases. Furthermore, the emission noises are also caused by the sputtering of the emitter by ionized gases originated from the residual gases [5]. In the new cold FEG, the space around the tip was evacuated with Non Evaporable Getter pumps (SAES Getters) and a $200 \mathrm{~L} / \mathrm{s}$ noble ion pump to decrease the residual gases [6]. The vacuum around the emitter reached well below $5 \times 10^{-9} \mathrm{~Pa}$. The high vacuum reduces the rate of adsorption of and sputtering by the residual gases so that the stable emission is attained. Figure 2 shows the change in the probe current and the emission current with time starting just after flashing. The stability of the probe current is much better than that of the emission current. The probe current is the beam current at the specimen, whereas the emission current is the total current emitted from the emitter. Obviously the probe current is more important for the performance of electron microscopes. The probe current gradually decreases $10 \%$ for the first 5 hours. This change was stable enough to start taking images and making analysis just after flashing.

Figure 3 shows the energy spread in the cold FEG with that in a Schottky gun. The FWHMs of the energy spreads in the cold FEG were $0.51 \mathrm{eV}$ and $0.26 \mathrm{eV}$ for high and low currents, respectively. They were substantially smaller than $0.7 \mathrm{eV}$ in the Schottky gun. These small energy spread improved the STEM resolution. Figure 4(a) shows a high resolution high angle annular dark field (HAADF) image of $\mathrm{GaN}(211)$ taken at $200 \mathrm{kV}$, Fig. $4 \mathrm{~b}$ is a line profile of the indicated area in Fig. 4(a) and Fig. 4(c) is the FFT pattern of Fig. 4(a). As shown in Figs. 4(a) and 4(b), 63 pm spacing between Ga-to-Ga dumbbell was well resolved in the image. As shown in Fig. 4(c), the information less than $63 \mathrm{pm}$ was transferred to the image.

The newly developed cold FEG with good stability made possible sub-Angstrom STEM imaging and high energy resolution analysis better than $0.3 \mathrm{eV}$ with JEM-ARM200F. 


\section{References}

[1] H. Sawada . et al., J. Electron Microsc. 54 (2005) 123.

[2] R. Erni, et al., PRL 102 (2009) 096101.

[3] E. Okunishi, et al., Microsc. Microanal. 12(Suppl 2) (2006) 1150.

[4] M. Bosman, et al., PRL 99 (2007) 086102.

[5] K. S. Yeong, and J. T. L. Thong, J. Appl. Phys. 99, 2006, 104903-1

[6] T. Tomita, et al., Microsc. Microanal. 15(Suppl 2), 2009, 1084.

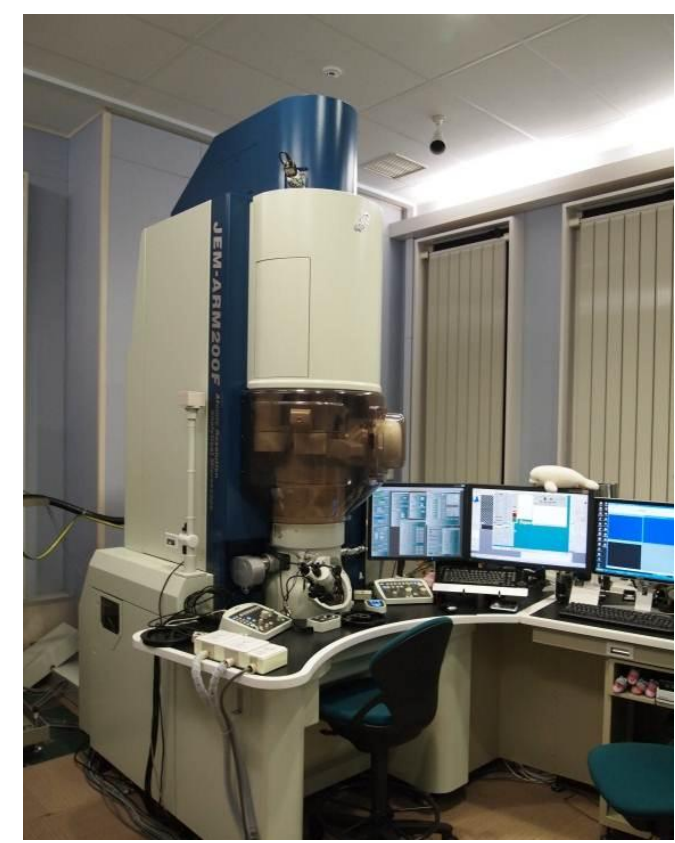

FIG. 1. The appearance of JEMARM200F with the cold FEG.

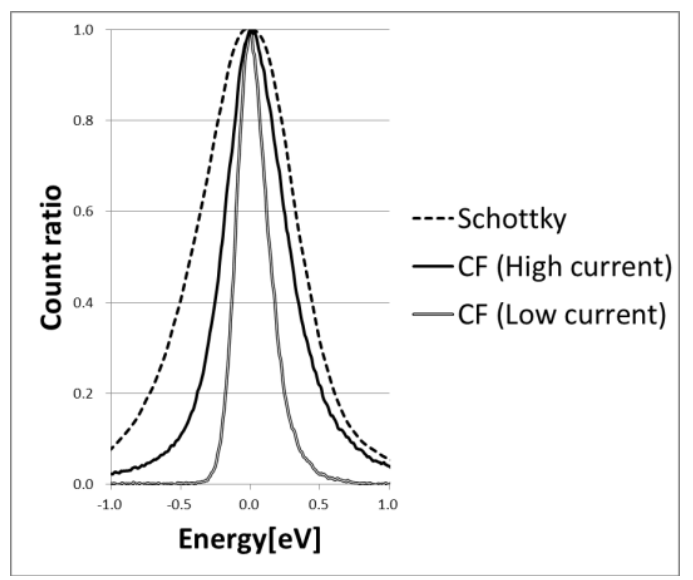

FIG. 3. The energy spread in the cold FE and Schottky gun.
FIG. 2. The change in the probe and emission current after flashing.

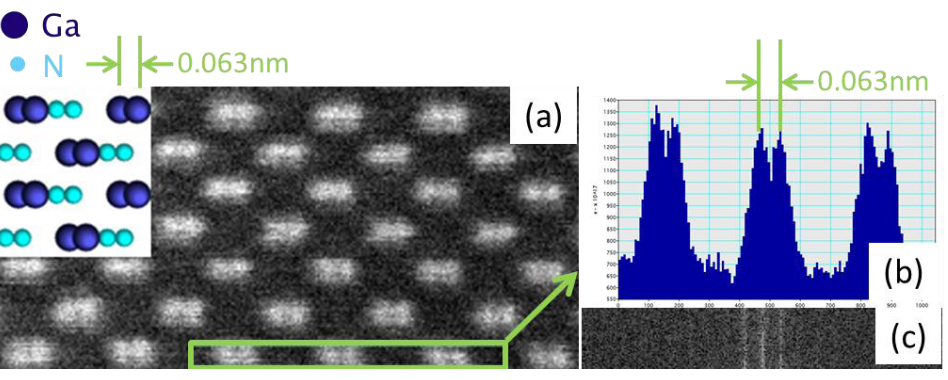

